\title{
Global existence of a shock for the supersonic flow past a curved wedge
}

\author{
Yin Huicheng \\ (Department of Mathematics, Nanjing University, Nanjing 210093, P.R.China) \\ (Institute of Mathematical Sciences, CUHK, Shatin, N.T., Hong Kong) \\ Email address: huicheng@nju.edu.cn
}

\begin{abstract}
This note is devoted to the study on the global existence of a shock wave for the supersonic flow past a curved wedge. When the curved wedge is a small perturbation of a straight wedge and the angle of the wedge is less than some critical value, we show that a shock attached at the wedge will exist globally.
\end{abstract}

\section{§1. Introduction}

This note is concerned with the global existence problem of a shock wave for the stationary supersonic flow past an infinite curved wedge. When the wedge is straight (that is, it is formed by two planes), such a problem has been solved well in the book [1] by the shock polar. For the curved wedge, if the direction of the supersonic coming flow is perpendicular to the edge of the wedge and the angle of the wedge is less than a critical value, which is determined by the velocity, density and adiabatic exponent of coming flow, then the local existence of a shock attached at the edge is proved in [2-4]. Besides, the author in [5] established the global existence of a weak solution by use of the Glimm's scheme for the isentropic steady supersonic flow past a 2-D infinite curved wedge with a sharp angle. Our main interest is on the structure of the global weak solution of such a problem. The aim of this paper is to show the global existence of a shock as observed in physical experiments, moreover we will remove the restriction on the sharp angle in [5].

Now let's give a detailed description of our main result. Suppose that the stationary inviscid supersonic flow past a 2-D wedge is governed by the steady Euler system. Under the assumptions that the flow is isentropic and irrotational, the system can be written as

$$
\left\{\begin{array}{l}
\partial_{x}(\rho u)+\partial_{y}(\rho v)=0 \\
\partial_{x}\left(\rho u^{2}\right)+\partial_{y}(\rho u v)+\partial_{x} P(\rho)=0 \\
\partial_{x}(\rho u v)+\partial_{y}\left(\rho v^{2}\right)+\partial_{y} P(\rho)=0
\end{array}\right.
$$

where $\rho,(u, v)$ and $P(\rho)$ represent the density, the velocity and the pressure respectively.

Suppose that there is a uniform supersonic flow $(u, v)=\left(q_{0}, 0\right)$ with constant density $\rho_{0}>0$, which comes from minus infinity. The flow hits a 2-D wedge along the direction perpendicular to the edge of the wedge. When the angle of the wedge is less than a critical value $b_{*}$, then the flow on the upper part and on the lower part can be determined independently. Without loss of generality, in this paper we only take into account the upper part of the wedge, whose equation in $x \geq 0$ is described by a smooth function $y=b(x)$ with $b(0)=0$ and $b^{\prime}(0)=b_{0}>0$.

\footnotetext{
* The author was supported by the National Natural Science Foundation of China.
} 
If $b_{0}<b_{*}$, then there will be a shock attached at the point $(0,0)$. Denote by $y=\chi(x)$ the equation of the shock front for $x \geq 0$, here $\chi(0)=0$. On the wedge and the shock front, the following boundary conditions should be satisfied

$$
u b^{\prime}(x)-v=0, \quad \text { on } \quad y=b(x)
$$

and

$$
\left\{\begin{array}{l}
{[\rho u] \chi^{\prime}(x)-[\rho v]=0,} \\
{\left[\rho u^{2}+P(\rho)\right] \chi^{\prime}(x)-[\rho u v]=0, \quad \text { on } \quad y=\chi(x)} \\
{[\rho u v] \chi^{\prime}(x)-\left[\rho v^{2}+P(\rho)\right]=0,}
\end{array}\right.
$$

where the system (1.3) comes from the Rankine-Hugoniot conditions.

Since the flow is irrotational, then one can deduce from (1.1) that

$$
\nabla_{x, y}\left(\frac{1}{2}\left(|u|^{2}+|v|^{2}\right)+h(\rho)\right)=0
$$

where $h(\rho)$ is the specific enthalpy, and $h^{\prime}(\rho)=\frac{c^{2}(\rho)}{\rho}, c^{2}(\rho)=P^{\prime}(\rho)>0$ for $\rho>0$.

The integration of (1.4) leads to the Bernoulli's law

$$
\frac{1}{2}\left(u^{2}+v^{2}\right)+h(\rho)=\frac{1}{2} q_{0}^{2}+h\left(\rho_{0}\right) \equiv C_{0}
$$

Then by the implicit function theorem we have

$$
\rho=h^{-1}\left(C_{0}-\frac{1}{2}\left(u^{2}+v^{2}\right)\right) \equiv H\left(u^{2}+v^{2}\right)
$$

Consequently, substituting (1.6) into the first equation in (1.1) we can reduce the system (1.1) into the following $2 \times 2$ system on $(u, v)$

$$
\left\{\begin{array}{l}
\partial_{x}(H u)+\partial_{y}(H v)=0 \\
\partial_{x} v-\partial_{y} u=0
\end{array}\right.
$$

It is easy to verify that (1.7) is strictly hyperbolic with respect to $x$-direction if $u>c(\rho)$. Indeed, for $u>c(\rho),(1.7)$ has two distinct real eigenvalues $\lambda_{1}(u, v)=\frac{u v-c(\rho) \sqrt{u^{2}+v^{2}-c^{2}(\rho)}}{u^{2}-c^{2}(\rho)}$ and $\lambda_{2}(u, v)=\frac{u v+c(\rho) \sqrt{u^{2}+v^{2}-c^{2}(\rho)}}{u^{2}-c^{2}(\rho)}$.

Furthermore, by (1.3) we obtain the following free boundary conditions on $y=\chi(x)$

$$
\left\{\begin{array}{l}
{[H u] \chi^{\prime}(x)-[H v]=0} \\
{[v] \chi^{\prime}(x)+[u]=0}
\end{array}\right.
$$

The main conclusion in this paper can be stated as:

Theorem 1.1. Suppose that the upper part of a curved wedge is given by $y=b(x)$, which satisfies

$$
\begin{aligned}
& b(0)=0, \quad b^{\prime}(0)=b_{0} \\
& \left|(1+x)^{k} \frac{d^{k}}{d x^{k}}\left(b(x)-b_{0} x\right)\right| \leq \varepsilon_{0} \quad \text { for } \quad 0 \leq k \leq 2, \quad x \geq 0,
\end{aligned}
$$


Assume that a uniform supersonic flow parallel to the $x$-axis comes from minus infinity with velocity $\left(q_{0}, 0\right)$, density $\rho_{0}>0$ satisfying $q_{0}>c\left(\rho_{0}\right)$. Additionally, $b_{0}>0$ is assumed to be less than $b_{*}$. Then (1.7) with the boundary conditions (1.2) and (1.8) admits a global weak entropy solution with a shock attached at the origin. Moreover, the location of the shock front and the flow field between the shock and the wedge tend to the corresponding ones for the flow past the unperturbed straight wedge $y=b_{0} x$ with the decay rate $(1+x)^{-\delta_{0}}$, here $\delta_{0}$ is some constant independent of $\varepsilon_{0}$.

Remark 1.1. We emphasize that there are no other discontinuities in our solution besides the main curved shock. This is contrast with the results in [5]. The condition (1.9) comes from the local existence theorem in [2-4] and the condition (1.10) especially gives a restriction on the perturbation of the wedge for large $x$. Our result demonstrates that the shock which produced by the supersonic flow past a wedge is structurally stable in a global sense.

Remark 1.2. Since the shock is weak, then the isentropic equation (1.7) actually gives a good approximation for the general 2-D stationary compressible Euler equations (see [6-7]).

In order to prove Theorem 1.1, we need to establish some global uniform estimates for the solution and its derivatives by the characteristics method. This is also the spirit of the results in [8]. To obtain the needed uniform estimates, we have to give some detailed computations in terms of the physical properties of shock wave as well as the boundary conditions (1.2) and $(1.8)$.

This note is organized as follows: In section 2, we give a reformulation of problem (1.7). In section 3, we complete the proof of Theorem 1.1.

\section{$\S 2$. The reformulation of main problem}

In this section, we firstly recall some basic properties for the supersonic flow past the straight wedge $y=b_{0} x$ in $x \geq 0$. On the left of shock wave, the flow has the density $\rho_{0}$ and the velocity $\left(q_{0}, 0\right)$, between the shock and the fixed boundary, the constant solution $\left(\rho_{+}, u_{+}, v_{+}\right)$can be obtained by use of the shock polar curve (see [1]). Here and below, $u_{+}>c_{+}=c\left(\rho_{+}\right)$will be assumed.

Now we list some properties on the solution $\left(\rho_{+}, u_{+} \cdot v_{+}\right)$. Denote by $y=s_{0} x$ the equation of shock wave, then the Rankine-Hugoniot condition (1.8) implies

$$
\left\{\begin{array}{l}
\rho_{+} v_{+}-s_{0}\left(\rho_{+} u_{+}-\rho_{0} q_{0}\right)=0 \\
u_{+}+s_{0} v_{+}-q_{0}=0
\end{array}\right.
$$

by use of the fixed boundary condition $v_{+}=b_{0} u_{+}$in (1.2), one can get

$$
\left\{\begin{array}{l}
u_{+}=\frac{q_{0}}{1+s_{0} b_{0}} \\
v_{+}=\frac{b_{0} q_{0}}{1+s_{0} b_{0}} \\
\rho_{+}=\frac{s_{0} \rho_{0}\left(1+s_{0} b_{0}\right)}{s_{0}-b_{0}}
\end{array}\right.
$$

Meanwhile, $s_{0}$ is determined from the Bernoulli's law (1.5), namely, $s_{0}$ satisfies

$$
\frac{\left(1+b_{0}^{2}\right) q_{0}^{2}}{2\left(1+s_{0} b_{0}\right)^{2}}+h\left(\frac{s_{0} \rho_{0}\left(1+s_{0} b_{0}\right)}{s_{0}-b_{0}}\right) \equiv C_{0}
$$


From the book [1], for $u_{+}>c_{+}$, we know that the algebraic equation (2.3) on $s_{0}$ has three distinct real roots when $b_{0}$ is less than the critical angle $b_{*}$. Physically, $s_{0}$ takes the intermediate value, it corresponds to the formation of weak oblique shock. Since the shock is weak, without loss of generality we will assume

$$
v_{+} \leq 2 c\left(\rho_{0}\right)
$$

Additionally, the P.Lax's entropy condition on 2-shock holds:

$$
\left\{\begin{array}{l}
\lambda_{1}\left(u_{+}, v_{+}\right)<s_{0}<\lambda_{2}\left(u_{+}, v_{+}\right) \\
\frac{c\left(\rho_{0}\right)}{\sqrt{q_{0}^{2}-c^{2}\left(\rho_{0}\right)}}<s_{0}
\end{array}\right.
$$

From (2.5), one easily gets

$$
\frac{s_{0} u_{+}-v_{+}}{\sqrt{1+s_{0}^{2}}}<c\left(\rho_{+}\right)
$$

The physical sense of (2.6) is: across the shock, the normal velocity of supersonic flow becomes subsonic.

Based on the above preparations, now we can reformulate the nonlinear problem (1.7) since the solution of (1.7) is actually a small perturbation of $\left(\rho_{+}, u_{+}, v_{+}\right)$.

Introducing two Riemann invariants as follows

$$
\begin{aligned}
& w_{1}=\operatorname{arctg} \frac{v}{u}+F\left(\sqrt{u^{2}+v^{2}}\right)-\operatorname{arctg} b_{0} \\
& w_{2}=\operatorname{arctg} \frac{v}{u}-F\left(\sqrt{u^{2}+v^{2}}\right)-\operatorname{arctg} b_{0}
\end{aligned}
$$

where $F^{\prime}(q)=\frac{\sqrt{q^{2}-c^{2}\left(H\left(q^{2}\right)\right)}}{q c\left(H\left(q^{2}\right)\right)}$ and $F\left(\frac{\sqrt{1+b_{0}^{2}}}{1+s_{0} b_{0}} q_{0}\right)=0$, then we can change (1.7) into a $2 \times 2$ system on $\left(w_{1}, w_{2}\right)$ :

$$
\left\{\begin{array}{l}
\partial_{x} w_{1}+\lambda_{1}(w) \partial_{y} w_{1}=0 \\
\partial_{x} w_{2}+\lambda_{2}(w) \partial_{y} w_{2}=0
\end{array}\right.
$$

where $\lambda_{i}(w)=\lambda_{i}\left(u\left(w_{1}, w_{2}\right), v\left(w_{1}, w_{2}\right)\right), i=1,2, u\left(w_{1}, w_{2}\right)$ and $v\left(w_{1}, w_{2}\right)$ are the inverse functions of $(2.7)$, moreover $u(0,0)=u_{+}$and $v(0,0)=v_{+}$.

Correspondingly, the boundary condition (1.8) on $y=\chi(x)$ becomes

$$
\left\{\begin{array}{l}
H\left(w_{1}, w_{2}\right) v^{2}\left(w_{1}, w_{2}\right)+\left(H\left(w_{1}, w_{2}\right) u\left(w_{1}, w_{2}\right)-\rho_{0} q_{0}\right)\left(u\left(w_{1}, w_{2}\right)-q_{0}\right)=0 \\
\chi^{\prime}(x)=\frac{q_{0}-u\left(w_{1}, w_{2}\right)}{v\left(w_{1}, w_{2}\right)}
\end{array}\right.
$$

and the fixed boundary condition (1.2) is

$$
u\left(w_{1}, w_{2}\right) b^{\prime}(x)-v\left(w_{1}, w_{2}\right)=0 \quad \text { on } \quad y=b(x)
$$

By Taylor's formula and the implicit function theorem, for the small $\left|w_{1}\right|$ and $\left|w_{2}\right|,(2.9)$ can be rewritten as

$$
w_{1}=A w_{2}+f_{1}\left(w_{2}\right) \quad \text { on } \quad y=\chi(x)
$$


where

$$
\begin{aligned}
& A=\frac{c_{+} u_{+}+v_{+} \sqrt{u_{+}^{2}+v_{+}^{2}-c_{+}^{2}}}{c_{+} v_{+}+u_{+} \sqrt{u_{+}^{2}+v_{+}^{2}-c_{+}^{2}}} \frac{m_{1}-m_{2} \lambda_{2}\left(u_{+}, v_{+}\right)}{m_{1}-m_{2} \lambda_{1}\left(u_{+}, v_{+}\right)} \\
& m_{1}=-\frac{\rho_{+}}{c_{+}^{2}} v_{+}^{3}+2 \rho_{+} v_{+}+\frac{\rho_{+}}{c_{+}^{2}} u_{+} v_{+}\left(q_{0}-u_{+}\right) \\
& m_{2}=-\frac{\rho_{+}}{c_{+}^{2}} u_{+} v_{+}^{2}+\left(\frac{\rho_{+}}{c_{+}^{2}} u_{+}^{2}-\rho_{+}\right)\left(q_{0}-u_{+}\right)+\left(\rho_{+} u_{+}-\rho_{0} q_{0}\right)
\end{aligned}
$$

and $f_{1}(0)=f_{1}^{\prime}(0)=0, f_{1}(w)$ is smooth.

Similarly, from the boundary condition (2.11), one can derive

$$
w_{2}=B w_{1}+f_{2}\left(b^{\prime}(x)-b_{0}, w_{1}\right) \quad \text { on } \quad y=b(x)
$$

where

$$
B=\frac{c_{+} v_{+}+u_{+} \sqrt{u_{+}^{2}+v_{+}^{2}-c_{+}^{2}}}{c_{+} u_{+}+v_{+} \sqrt{u_{+}^{2}+v_{+}^{2}-c_{+}^{2}}} \frac{1+b_{0} \lambda_{1}\left(u_{+}, v_{+}\right)}{1+b_{0} \lambda_{2}\left(u_{+}, v_{+}\right)}
$$

and $f_{2}(0,0)=\left(\partial_{2} f_{2}\right)(0,0)=0, f_{2}$ is smooth.

Hence in order to prove Theorem 1.1, by the local existence of solution in [2-4], we only need to solve the problem $(2.8),(2.10),(2.12),(2.13)$ with the small initial data $\left.w_{i}(x, y)\right|_{x=1}(i=1,2)$ and $\left.\left(\chi(x)-s_{0} x\right)\right|_{x=1}$ in the domain $\{(x, y): x \geq 1, b(x) \leq y \leq \chi(x)\}$. The smallness means

$$
\sum_{|\alpha| \leq 1} \sup _{b(1) \leq y \leq \chi(1)}\left|\nabla_{x, y}^{\alpha} w_{i}(1, y)\right| \leq C \varepsilon_{0}, \quad\left|\chi(1)-s_{0}\right| \leq C \varepsilon_{0}, \quad\left|\chi^{\prime}(1)-s_{0}\right| \leq C \varepsilon_{0}
$$

where $\varepsilon_{0}$ is given in Theorem 1.1. We note that (2.14) can be derived from the result on the local existence and stability in [2-4].

\section{$\S 3$. The proof of Theorem 1.1}

In order to prove Theorem 1.1, as the first step, we need to verify that the condition $|A B|<1$ holds, where the constants $A$ and $B$ appear in (2.12) and (2.13) respectively. The condition $|A B|<1$ will lead to the uniform bound of solution to (2.8) in the domain $\{(x, y): x \geq 1, b(x) \leq$ $y \leq \chi(x)\}$.

Lemma 3.1. In (2.12), $m_{1}>0$ and $m_{2}>0$.

Proof. In terms of the expressions of $u_{+}, v_{+}$in (2.2), we have

$m_{1}=\frac{\rho_{+} v_{+}}{c_{+}^{2}}\left(2 c_{+}^{2}-v_{+}^{2}+u_{+}\left(q_{0}-u_{+}\right)\right)>\frac{\rho_{+} v_{+}}{c_{+}^{2}}\left(-v_{+}^{2}+u_{+}\left(q_{0}-u_{+}\right)\right)=\frac{\rho_{+} v_{+}}{c_{+}^{2}} \frac{b_{0} q_{0}^{2}\left(s_{0}-b_{0}\right)}{\left(1+s_{0} b_{0}\right)^{2}}>0$

Similarly, we can get

$$
\begin{aligned}
& m_{2}=\frac{\rho_{+}}{c_{+}^{2}}\left(\left(u_{+}^{2}-c_{+}^{2}\right)\left(q_{0}-u_{+}\right)-u_{+} v_{+}^{2}\right)+\left(\rho_{+} u_{+}-\rho_{0} q_{0}\right) \\
&=\frac{b_{0} \rho_{0} q_{0}}{\left(s_{0}-b_{0}\right) c_{+}^{2}}\left(s_{0}\left(s_{0}-b_{0}\right) u_{+}^{2}+\left(1-s_{0}^{2}\right) c_{+}^{2}\right) \\
& 5
\end{aligned}
$$


If $s_{0} \leq 1$, obviously one has $m_{2}>0$.

If $s_{0} \geq 1$, then the entropy condition (2.6) leads to

$$
m_{2}>\frac{b_{0} \rho_{0} q_{0}}{\left(s_{0}-b_{0}\right) c_{+}^{2}}\left(s_{0}\left(s_{0}-b_{0}\right) u_{+}^{2}+\left(1-s_{0}^{2}\right) \frac{\left(s_{0}-b_{0}\right)^{2} u_{+}^{2}}{1+s_{0}^{2}}\right)>\frac{b_{0}^{2} \rho_{0} q_{0} u_{+}^{2}}{c_{+}^{2}}>0
$$

Hence Lemma 3.1 holds.

Lemma 3.2. 1). $1+b_{0} \lambda_{1}\left(u_{+}, v_{+}\right)>0$.

$2)$. Under the condition (2.4), $|A B|<1$ holds.

Proof. 1). By a direct computation, one has

$1+b_{0} \lambda_{1}\left(u_{+}, v_{+}\right)=\frac{u_{+}^{2}-c_{+}^{2}+b_{0} u_{+} v_{+}-b_{0} c_{+} \sqrt{u_{+}^{2}+v_{+}^{2}-c_{+}^{2}}}{u_{+}^{2}-c_{+}^{2}}=\frac{\left(1+b_{0}^{2}\right) \sqrt{u_{+}^{2}+v_{+}^{2}-c_{+}^{2}}}{\sqrt{u_{+}^{2}+v_{+}^{2}-c_{+}^{2}}+b_{0} c_{+}}>0$

2). Firstly, under the condition (2.4), we assert

$$
m_{1}-m_{2} \lambda_{1}\left(u_{+}, v_{+}\right)>0
$$

Indeed, if $v_{+} \leq c_{+}$, then $\lambda_{1}\left(u_{+}, v_{+}\right) \leq 0$, so by lemma 3.1, (3.2) obviously holds. If $v_{+} \geq c_{+}$, then $\lambda_{1}\left(u_{+}, v_{+}\right) \geq 0$, moreover by $(2.4)$ we have $v_{+}<2 c_{+}$. A direct computation yields

$$
\begin{aligned}
m_{1}- & m_{2} \lambda_{1}\left(u_{+}, v_{+}\right)=\frac{\rho_{+}}{c_{+}^{2}}\left(2 c_{+}^{2} v_{+}-v_{+}^{3}+c_{+}\left(q_{0}-u_{+}\right) \sqrt{u_{+}^{2}+v_{+}^{2}-c_{+}^{2}}+u_{+} v_{+}^{2} \lambda_{1}\left(u_{+}, v_{+}\right)\right) \\
& -\left(\rho_{+} u_{+}-\rho_{0} q_{0}\right) \lambda_{1}\left(u_{+}, v_{+}\right) \\
& >\frac{\rho_{+} v_{+}}{c_{+}^{2}}\left(2 c_{+}^{2}-v_{+}^{2}+c_{+} v_{+}\right)+\rho_{+} v_{+}\left(\frac{u_{+} v_{+}}{c_{+}^{2}}-\frac{1}{s_{0}}\right) \lambda_{1}\left(u_{+}, v_{+}\right) \\
& >\frac{\rho_{+} v_{+}}{c_{+}^{2}}\left(c_{+}+v_{+}\right)\left(2 c_{+}-v_{+}\right)+\rho_{+} v_{+}\left(\frac{1}{b_{0}}-\frac{1}{s_{0}}\right) \lambda_{1}\left(u_{+}, v_{+}\right)>0
\end{aligned}
$$

Now we show $|A B|<1$.

If $m_{1}-m_{2} \lambda_{2}\left(u_{+}, v_{+}\right) \geq 0$, by (3.1) and Lemma 3.1 then

$$
1-|A B|=\frac{\left(m_{2}+b_{0} m_{1}\right)\left(\lambda_{2}\left(u_{+}, v_{+}\right)-\lambda_{1}\left(u_{+}, v_{+}\right)\right)}{\left(m_{1}-m_{2} \lambda_{1}\left(u_{+}, v_{+}\right)\right)\left(1+b_{0} \lambda_{2}\left(u_{+}, v_{+}\right)\right)}>0
$$


If $m_{1}-m_{2} \lambda_{2}\left(u_{+}, v_{+}\right)<0$, by (3.1) and Lemma 3.1 then

$$
\begin{aligned}
1-|A B|= & \frac{2 m_{1}-2 b_{0} m_{2} \lambda_{1}\left(u_{+}, v_{+}\right) \lambda_{2}\left(u_{+}, v_{+}\right)+\left(b_{0} m_{1}-m_{2}\right)\left(\lambda_{1}\left(u_{+}, v_{+}\right)+\lambda_{2}\left(u_{+}, v_{+}\right)\right)}{\left(m_{1}-m_{2} \lambda_{1}\left(u_{+}, v_{+}\right)\right)\left(1+b_{0} \lambda_{2}\left(u_{+}, v_{+}\right)\right)} \\
= & \frac{2 \rho_{+} v_{+}}{c_{+}^{2}\left(u_{+}^{2}-c_{+}^{2}\right)\left(m_{1}-m_{2} \lambda_{1}\left(u_{+}, v_{+}\right)\right)\left(1+b_{0} \lambda_{2}\left(u_{+}, v_{+}\right)\right)}\left\{\left[2 c_{+}^{2}+\left(s_{0} b_{0}-b_{0}^{2}\right) u_{+}^{2}\right]\left(u_{+}^{2}-c_{+}^{2}\right)\right. \\
& \quad-\left[\left(1-b_{0}^{2}\right)\left(s_{0}-b_{0}\right) u_{+}^{2}-\left(s_{0}+2 b_{0}-\frac{1}{s_{0}}\right) c_{+}^{2}\right] b_{0} u_{+}^{2}-\left[b_{0}\left(s_{0}-b_{0}\right) u_{+}^{2}\right. \\
& \left.\left.\quad-b_{0}\left(s_{0}-\frac{1}{s_{0}}\right) c_{+}^{2}\right]\left(b_{0}^{2} u_{+}^{2}-c_{+}^{2}\right)\right\} \\
= & \frac{2 \rho_{+} v_{+}}{\left(u_{+}^{2}-c_{+}^{2}\right)\left(m_{1}-m_{2} \lambda_{1}\left(u_{+}, v_{+}\right)\right)\left(1+b_{0} \lambda_{2}\left(u_{+}, v_{+}\right)\right)}\left\{\left[2+b_{0}\left(s_{0}+2 b_{0}-\frac{1}{s_{0}}\right)\right.\right. \\
& \left.\left.\quad+b_{0}^{3}\left(s_{0}-\frac{1}{s_{0}}\right)\right] u_{+}^{2}-\left[2+b_{0}\left(s_{0}-\frac{1}{s_{0}}\right)\right] c_{+}^{2}\right\} \\
> & \frac{2 b_{0}^{2} \rho_{+} v_{+} u_{+}^{2}}{\left(u_{+}^{2}-c_{+}^{2}\right)\left(m_{1}-m_{2} \lambda_{1}\left(u_{+}, v_{+}\right)\right)\left(1+b_{0} \lambda_{2}\left(u_{+}, v_{+}\right)\right)}\left(1+s_{0} b_{0}\right)>0
\end{aligned}
$$

Therefore $|A B|<1$ holds.

To prove Theorem 1.1, now we give a uniform estimate on $w$ and its derivatives.

Lemma 3.3. Set $D_{T}=\{1 \leq x \leq T, b(x) \leq y \leq \chi(x)\}$ for any $T>1$. If $w \in C^{2}\left(D_{T}\right)$ satisfies (2.8)-(2.11) and (2.14), then there exist two positive constants $C_{0}$ and $\tilde{C}_{0}$ independent of $\varepsilon_{0}$ and $T$, such that $\left|\partial_{x, y}^{\alpha} w_{i}\right| \leq \frac{C_{0} \varepsilon_{0}}{(1+x)^{\delta_{0}+|\alpha|}}$ in $D_{T}$ for $|\alpha| \leq 1, i=1,2$, and $\left|\partial_{x}^{j}\left(\chi^{\prime}(x)-s_{0}\right)\right| \leq \frac{\tilde{C}_{0} \varepsilon_{0}}{(1+x)^{\delta_{0}+j}}$ in $[1, T]$ when $0 \leq j \leq 1$, here $\delta_{0}>0$ is an appropriate constant.

Proof. We shall use the reflected characteristics method to obtain the needed estimates (such a method has been used in Lemma 2.1 of Chapter 5 of [8]). By the local existence result of solution in [2-4] and the continuity induction, we only need to prove

For some constants $C_{0}>0,0<\delta_{0}<1$, if $\left|\partial_{x, y}^{\alpha} w_{i}\right| \leq \frac{C_{0} \varepsilon_{0}}{(1+x)^{\delta_{0}+|\alpha|}}$ in $D_{T}$ for $|\alpha| \leq 1, i=1,2$, then there exist two positive constants $C_{0}^{\prime}<C_{0}$ and $\tilde{C}_{0}$ such that $\left|\partial_{x, y}^{\alpha} w_{i}\right| \leq \frac{C_{0}^{\prime} \varepsilon_{0}}{(1+x)^{\delta_{0}+|\alpha|}}$ in $D_{T}$ for $|\alpha| \leq 1$, and $\left|\partial_{x}^{j}\left(\chi^{\prime}(x)-s_{0}\right)\right| \leq \frac{\tilde{C}_{0} \varepsilon_{0}}{(1+x)^{\delta_{0}+j}}$ in $[1, T]$ when $0 \leq j \leq 1$.

Below we denote by $C$ various strictly positive constants independent of $\varepsilon_{0}$ and $T$. If $(x, y) \in$ $D_{T}, x<T$, we shall denote by $\gamma_{j}^{-}(t, x, y)(j=1,2)$ the backward $\mathrm{j}$-th characteristic curve pass the point $(x, y)$, namely

$$
\left\{\begin{array}{l}
\frac{d \gamma_{j}^{-}(t, x, y)}{d t}=\lambda_{j}\left(w\left(t, \gamma_{j}^{-}(t, x, y)\right)\right), \quad t \leq x \\
\left.\gamma_{j}^{-}(t, x, y)\right|_{t=x}=y
\end{array}\right.
$$

By the assumptions in (3.2), one has

$$
\left|\frac{d \gamma_{j}^{-}(t, x, y)}{d t}-\lambda_{j}\left(u_{+}, v_{+}\right)\right| \leq \frac{C \varepsilon_{0}}{(1+x)^{\delta_{0}}} \quad \text { in } \quad D_{T}
$$


If $\left\{\left(t, \gamma_{1}^{-}(t, x, y)\right)\right\} \cap\{(t, z): z=\chi(t)\}=\left(\Gamma_{1}(x, y), \xi_{1}(x, y)\right),\left\{\left(t, \gamma_{2}^{-}(t, x, y)\right)\right\} \cap\{(t, z): z=$ $b(t)\}=\left(\Gamma_{2}(x, y), \xi_{2}(x, y)\right)$, then from the system $(2.8)$ we have

$$
w_{i}(x, y)=w_{i}\left(\Gamma_{i}(x, y), \xi_{i}(x, y)\right)
$$

If $\left\{\left(t, \gamma_{1}^{-}\left(t, \Gamma_{2}(x, y), \xi_{2}(x, y)\right)\right\} \cap\{(t, z): z=\chi(t)\}=\left(\pi_{1}(x, y), \eta_{1}(x, y)\right)\right.$ and $\left\{\left(t, \gamma_{2}^{-}\left(t, \Gamma_{1}(x, y)\right.\right.\right.$, $\left.\left.\xi_{1}(x, y)\right)\right\} \cap\{(t, z): z=b(t)\}=\left(\pi_{2}(x, y), \eta_{2}(x, y)\right)$, then by use of the characteristics method and the boundary conditions (2.12) and (2.13) we get

$$
\begin{aligned}
& \left|w_{2}(x, y)\right|=\left|B w_{1}\left(\Gamma_{2}(x, y), \xi_{2}(x, y)\right)+f_{2}\left(b^{\prime}\left(\Gamma_{2}(x, y)\right)-b_{0}, w_{1}\left(\Gamma_{2}(x, y), \xi_{2}(x, y)\right)\right)\right| \\
& \quad=\left|B w_{1}\left(\pi_{1}(x, y), \eta_{1}(x, y)\right)+f_{2}\left(b^{\prime}\left(\Gamma_{2}(x, y)\right)-b_{0}, w_{1}\left(\pi_{1}(x, y), \eta_{1}(x, y)\right)\right)\right| \\
& \quad \leq\left|A B w_{2}\left(\pi_{1}(x, y), \eta_{1}(x, y)\right)\right|+\left|B f_{1}\left(w_{2}\left(\pi_{1}(x, y), \eta_{1}(x, y)\right)\right)\right| \\
& \quad+\mid f_{2}\left(b^{\prime}\left(\Gamma_{2}(x, y)\right)-b_{0}, A w_{2}\left(\left(\pi_{1}(x, y), \eta_{1}(x, y)\right)+f_{1}\left(w_{2}\left(\pi_{1}(x, y), \eta_{1}(x, y)\right)\right)\right) \mid\right.
\end{aligned}
$$

Noting that for small $\varepsilon_{0}$, one has

$$
\begin{aligned}
& d_{i}-C \varepsilon_{0} \leq \frac{\Gamma_{i}(x, y)}{x} \leq 1+c \varepsilon_{0} \\
& d_{1} d_{2}-C \varepsilon_{0} \leq \frac{\pi_{i}(x, y)}{x} \leq d_{i}+C \varepsilon_{0}
\end{aligned}
$$

where $d_{1}=\frac{b_{0}-\lambda_{1}\left(u_{+}, v_{+}\right)}{s_{0}-\lambda_{1}\left(u_{+}, v_{+}\right)}>0$ and $d_{2}=\frac{\lambda_{2}\left(u_{+}, v_{+}\right)-s_{0}}{\lambda_{2}\left(u_{+}, v_{+}\right)-b_{0}}>0$.

Assume $\left|f_{2}\left(b^{\prime}(x)-b_{0}, w_{2}\right)\right| \leq C_{1}\left|b^{\prime}(x)-b_{0}\right|$ for small $\left|w_{2}\right|$, then by assumption in (3.2) and by use of $(3.6)$ and $d_{1} d_{2}<1$, we obtain

$$
\left|w_{2}(x, y)\right| \leq\left(\frac{|A B|}{\left(d_{1} d_{2}\right)^{\delta_{0}}} C_{0}+C_{1}+C \varepsilon_{0}\right) \frac{\varepsilon_{0}}{(1+x)^{\delta_{0}}}
$$

Similarly, one can obtain

$$
\left|w_{1}(x, y)\right| \leq\left(\frac{|A B|}{\left(d_{1} d_{2}\right)^{\delta_{0}}} C_{0}+C \varepsilon_{0}\right) \frac{\varepsilon_{0}}{(1+x)^{\delta_{0}}}
$$

If $\left\{\left(t, \gamma_{1}^{-}(t, x, y)\right)\right\} \cap\{(t, z): z=\chi(t)\}=\emptyset$, or $\left\{\left(t, \gamma_{2}^{-}(t, x, y)\right)\right\} \cap\{(t, z): z=b(t)\}=\emptyset$, or $\left\{\left(t, \gamma_{1}^{-}\left(t, \Gamma_{2}(x, y), \xi_{2}(x, y)\right)\right)\right\} \cap\{(t, z): z=\chi(t)\}=\emptyset$, or $\left\{\left(t, \gamma_{2}^{-}\left(t, \Gamma_{1}(x, y), \xi_{1}(x, y)\right)\right)\right\} \cap\{(t, z):$ $z=b(t)\}=\emptyset$, then by (3.4), (2.5) and the initial data (2.14) we can conclude

$$
x \leq \bar{C},\left|w_{i}(x, y)\right| \leq \bar{C} \varepsilon_{0}
$$

where $\bar{C}$ is independent of $\varepsilon_{0}$.

Therefore, if we choose $0<\delta_{0}<1$ such that $\frac{|A B|}{\left(d_{1} d_{2}\right)^{\delta_{0}}}<1$, and set $C_{0}=\max \left\{\frac{2 C_{1}}{1-\frac{|A B|}{\left(d_{1} d_{2}\right)^{\delta_{0}}}}, 2 \bar{C}(1\right.$ $\left.+\bar{C})^{\delta_{0}}\right\}$ and $C_{0}^{\prime}=\max \left\{\frac{C_{1}\left(1+\frac{|A B|}{\left(d_{1} d_{2}\right)^{\delta_{0}}}\right)}{1-\frac{A B \mid}{\left(d_{1} d_{2}\right)^{\delta_{0}}}}, \bar{C}(1+\bar{C})^{\delta_{0}}\right\}$, then we know (3.2) holds for $|\alpha|=0$. 
At this time, one has

$$
\left|\chi^{\prime}(x)-s_{0}\right| \leq\left|\frac{u\left(w_{1}, w_{2}\right)-u_{+}}{v\left(w_{1}, w_{2}\right)}\right|+\left|\frac{\left(u_{+}-q_{0}\right)\left(v\left(w_{1}, w_{2}\right)-v_{+}\right)}{v_{+} v\left(w_{1}, w_{2}\right)}\right| \leq \frac{\tilde{C}_{0} \varepsilon_{0}}{(1+x)^{\delta_{0}}}
$$

Next we prove (3.2) for $|\alpha|=1$.

Denote by $Z=\partial_{x}+b^{\prime}(x) \partial_{y}$ the tangent vector field of boundary $y=b(x)$. Set $\bar{w}_{i}=Z w_{i}, i=$ 1,2 , then from $(2.8)$ we get

$$
\left\{\begin{array}{l}
\partial_{x} \bar{w}_{1}+\lambda_{1}(w) \partial_{y} \bar{w}_{1}=f_{1} \\
\partial_{x} \bar{w}_{2}+\lambda_{2}(w) \partial_{y} \bar{w}_{2}=f_{2}
\end{array}\right.
$$

where $f_{i}=b^{\prime \prime}(x) \partial_{y} w_{i}-\left(\partial_{w_{1}} \lambda_{i} \bar{w}_{1}+\partial_{w_{2}} \lambda_{i} \bar{w}_{2}\right) \partial_{y} w_{i}$.

From (2.13), one has

$$
\bar{w}_{2}=\left(B+\left(\partial_{2} f_{2}\right)\left(b^{\prime}(x)-b_{0}, w_{1}\right)\right) \bar{w}_{1}+\left(\partial_{1} f_{2}\right)\left(b^{\prime}(x)-b_{0}, w_{1}\right) b^{\prime \prime}(x)
$$

To get the boundary condition of $\bar{w}$ on $y=\chi(x)$, one should notice that the vector field $V=\partial_{x}+\chi^{\prime}(x) \partial_{y}$ tangent to $y=\chi(x)$ can be expressed as follows

$$
V=\frac{1}{\lambda_{i}(w)-b^{\prime}(x)}\left\{\left(\lambda_{i}(w)-\chi^{\prime}(x)\right) Z+\left(\chi^{\prime}(x)-b^{\prime}(x)\right)\left(\partial_{x}+\lambda_{i}(w) \partial_{y}\right)\right\}
$$

So on the shock $y=\chi(x)$, from (2.12) and (2.8) we have

$$
\bar{w}_{1}=\frac{\left(\lambda_{2}(w)-\chi^{\prime}(x)\left(b^{\prime}(x)-\lambda_{1}(w)\right)\right)}{\left(\lambda_{2}(w)-b^{\prime}(x)\right)\left(\chi^{\prime}(x)-\lambda_{1}(w)\right)}\left(A+f_{1}^{\prime}\left(w_{2}\right)\right) \bar{w}_{2}
$$

By the assumptions in Theorem 1.1 and (3.2), we have

$$
\left|f_{i}(x, y)\right| \leq C \varepsilon_{0}^{2}\left(\frac{1}{(1+x)^{3+\delta_{0}}}+\frac{1}{(1+x)^{2+2 \delta_{0}}}\right) \leq \frac{C \varepsilon_{0}^{2}}{(1+x)^{2+2 \delta_{0}}}
$$

Using the notations above, if $\gamma_{1}^{-}(t, x, y)$ and $\gamma_{2}^{-}(t, x, y)$ both intersect with fixed boundary and shock front, then by the characteristics method, (3.10) and the boundary conditions (3.8), (3.9), one has

$$
\begin{aligned}
& \left|\bar{w}_{2}(x, y)\right| \leq\left|\bar{w}_{2}\left(\Gamma_{2}(x, y), \xi_{2}(x, y)\right)\right|+\int_{x}^{\Gamma_{2}(x, y)} \frac{C \varepsilon_{0}^{2}}{(1+s)^{2+2 \delta_{0}}} d s \\
& \quad \leq\left|B \bar{w}_{1}\left(\Gamma_{2}(x, y), \xi_{2}(x, y)\right)\right|+\frac{C \varepsilon_{0}^{2}}{(1+x)^{1+2 \delta_{0}}} \\
& \quad \leq\left|B \bar{w}_{1}\left(\pi_{1}(x, y), \eta_{1}(x, y)\right)\right|+\frac{C \varepsilon_{0}^{2}}{(1+x)^{1+2 \delta_{0}}} \\
& \quad \leq|A B| d_{1} d_{2}\left|\bar{w}_{2}\left(\pi_{1}(x, y), \eta_{1}(x, y)\right)\right|+\frac{C \varepsilon_{0}^{2}}{(1+x)^{1+2 \delta_{0}}} \\
& \quad \leq \frac{|A B|}{\left(d_{1} d_{2}\right)^{\delta_{0}}} \frac{C_{0} \varepsilon_{0}}{(1+x)^{1+\delta_{0}}}+\frac{C \varepsilon_{0}^{2}}{(1+x)^{1+2 \delta_{0}}}
\end{aligned}
$$


Similarly, we have

$$
\left|\bar{w}_{1}(x, y)\right| \leq \frac{|A B|}{\left(d_{1} d_{2}\right)^{\delta_{0}}} \frac{C_{0} \varepsilon_{0}}{(1+x)^{1+\delta_{0}}}+\frac{C \varepsilon_{0}^{2}}{(1+x)^{1+2 \delta_{0}}}
$$

Noting $\partial_{x} w_{i}=\frac{\lambda_{i}(w) \bar{w}_{i}}{\lambda_{i}(w)-b^{\prime}(x)}, \partial_{y} w_{i}=-\frac{\bar{w}_{i}}{\lambda_{i}(w)-b^{\prime}(x)}$ and $\frac{|A B|}{\left(d_{1} d_{2}\right)^{\delta_{0}}}<1$, then similar to the proof on $w$ in the above we know that (3.2) also holds for $|\alpha|=1$.

Since $\chi^{\prime \prime}(x)=\sum_{i=1}^{2} \partial_{w_{i}}\left(\frac{q_{0}-u\left(w_{1}, w_{2}\right)}{v\left(w_{1}, w_{2}\right)}\right)\left(\partial_{x} w_{i}+\partial_{y} w_{i} \chi^{\prime}(x)\right)$ on $y=\chi(x)$, then by the estimates on $w_{i}$ and $\partial_{x} w_{i}, \partial_{y} w_{i}$, it is easy to conclude

$$
\left|\chi^{\prime \prime}(x)\right| \leq \frac{\tilde{C}_{0} \varepsilon_{0}}{(1+x)^{1+\delta_{0}}}
$$

Therefore (3.2) and Lemma 3.3 are proved.

Proof of Theorem 1.1. Since the local existence of the solution of (2.8)-(2.11) near the origin $(0,0)$ is achieved in [2-3], while for any given $x_{0}$, the solution of $(2.8)$ with the initial data given on $x=x_{0}$ as in (2.14) and the boundary conditions (2.9) -(2.11) in $\left[x_{0}, x_{0}+\frac{C}{\varepsilon_{0}}\right]$ can be obtained by use of the characteristics method. Therefore, by Lemma 3.3, we can get the smaller initial data of $w$ on $\frac{C}{\varepsilon_{0}}$, then the solution can be extended continuously to the whole domain.

Acknowledgments. The author would like to express his sincere thanks to Prof.Chen Shuxing and Prof.Xin Zhouping for their invaluable discussion. He also thanks the Institute of Mathematics in Potsdam University, where he worked on this paper during his visiting period.

\section{REFERENCES}

1. R.Courant, K.O. Friedrichs, Supersonic flow and shock waves, Springer, New York, 1976.

2. D.G.Schaeffer, Supersonic flow past a nearly straight wedge, Duke Math.J., 43, 637-670 (1976).

3. T.Li, On a free boundary problem, Chin.Ann.Math., 1, 351-358 (1980).

4. Shuxing Chen, Existence of local solution to supersonic flow past a three-dimensional wing, Adv.Appl.Math., 13, 273-304 (1992).

5. Yongqian Zhang, Global existence of steady supersonic potential flow past a curved wedge with a piecewise smooth boundary, SIAM J.Math. Anal. Vol.31, No.1, 166-183 (1999).

6. A.Majda, One perspective on open problems in multidimensional conservation laws, Multidimensional Hyperbolic Problems and Computation, Springer Verlag, IMA 29, 217-237 (1990).

7. C.S.Morawetz, Potential theory for regular and Mach reflection of a shock at a wedge, Comm. Pure Appl. Math., 47, 593-624 (1994).

8. Tatsien Li, Global classical solutions for quasilinear hyperbolic systems, Research in Applied Mathematics 34, Wiley, Masson, New York, Paris, 1994. 